\title{
High Species Diversity of the Family Dipterocarpaceae in Mursala Island, Indonesia
}

\author{
Iyan Robiansyah $^{\mathrm{a}}$, Arief Hamidi ${ }^{\mathrm{b}}$, Agusti Randi ${ }^{\mathrm{c}}$ \\ ${ }^{a}$ Research Center for Plant Conservation and Botanic Gardens, Indonesian Institute of Sciences (LIPI), Bogor, 16003 Indonesia. \\ E-mail: iyan.robiansyah@lipi.go.id \\ ${ }^{b}$ Fauna \& Flora International-Indonesia Programme, South Jakarta, 12450, Indonesia \\ E-mail: arief.hamidi@fauna-flora.org \\ ${ }^{c}$ Indonesian Forum for Threatened Trees, Bogor, 16003, West Java, Indonesia \\ Email: randiagusti@gmail.com
}

\begin{abstract}
Known to have a long history of timber extraction, Mursala Island on the west coast of North Sumatra Province is a small island home to the endemic tree Dipterocarpus cinereus and many other Dipterocarpaceae species. In the present study, distance sampling using line-transect was used to assess the species diversity and population status of Dipterocarpaceae species on the Island. A total of 26 Dipterocarpaceae species from 6 genera was identified from Mursala Island. The genus that was represented by the highest number of species was Shorea (13 species), followed by Vatica (5), Dipterocarpus (4), Hopea (2), Cotylelobium (1) and Dryobalanops (1). Among these, two Mursala Island endemic were identified: $D$. cinereus and $H$. bancana. Furthermore, according to IUCN Red List category, 11 species $(\mathbf{4 2 . 3 \%})$ were classified as critically endangered, four species $(15.4 \%)$ were of endangered, 5 species $(15.2 \%)$ were of vulnerable, one species was of near threatened, 2 species were of least concern and 3 species were not assessed yet. Based on distance analysis, the species with the highest individual density was $S$. multiflora $(21.72 \pm 10.96$ individual/ha), whereas species with the lowest density was $S$. johorensis $(0.06 \pm 0.01$ individual/ha). Two main threats for Dipterocarpaceae species in Mursala Island were observed during the survey: illegal logging and habitat conversion. Increasing the protection level of the island was recommended to conserve all the species of Dipterocarpaceae. Besides, providing alternative sources of living for the illegal logger through commercialization of non-timber forest products, ecotourism, and the sustainable fishery was recommended so that they could stop illegal logging activity.
\end{abstract}

Keywords_-Dipterocarpaceae; Dipterocarpus cinereus; distance sampling; endemic tree; Hopea bancana; Mursala Island.

\section{INTRODUCTION}

Dipterocarpaceae is one of the biggest tree family with >500 species in the world [1]-[3]. It is a pantropical family, distributed in the lowlands and hills of the tropics below $1800 \mathrm{~m}$ [3]. The family includes three subfamilies: Monotoideae in Africa and South America, Pakaraimoideae in South America, and Dipterocarpoideae in Asia [2]. The species from the last subfamily were mainly found in the Malesian region (ca. 386 species) [1].

In Indonesia, there were 238 species of Dipterocarpaceae or $62 \%$ of the Malesian species, with Kalimantan (200 species) and Sumatra (111 species) being the center of the diversity [1]. Most of these species are in lowland forests below $1500 \mathrm{~m}$, an area with a high rate of tree cutting and forest conversion. Consequently, a total of 151 species
(63.4\%) of Indonesian Dipterocarpaceae are included as threatened species by IUCN Red List [4].

Known to have a long history of timber extraction, Mursala Island on the west coast of North Sumatra Province is home to the endemic Dipterocarpus cinereus and many other Dipterocarpaceae species. Due to this extensive timber extraction, Ashton [5] declared that D. cinereus was extinct in the wild in 1998. A team from Bogor Botanic Gardens - LIPI, however, reported the rediscovery of the species in 2013 [6]. Additional explorations at the end of 2013 [7] and 2017 [8] have been conducted on the island confirming the present of the species. Besides, these explorations reported the presence of other 22 species of Dipterocarpaceae. This high richness of Dipterocarpaceae has made the island one of the hotspots for Dipterocarpaceae biodiversity.

As previous studies only covered small areas of the west part of Mursala Island, further studies that cover wider areas 
are needed to reveal the current status of $D$. cinereus and to assess Dipterocarpaceae diversity of the island. This information is crucial for conservation planning and actions of all these species as illegal cutting and timber extraction on the island are still ongoing at an alarming rate. Thus, in the present study, distance sampling using line-transect will be used to assess the Dipterocarpaceae population in Mursala Island. The objectives are to i) assess the diversity and distribution of Dipterocarpaceae in the island, ii) assess the population structure of all the species, iii) estimate the density of all the species based on distance sampling applied, and iv) identify all threats that may threaten all the Dipterocarpaceae species and their habitat.

\section{THE MATERIALS AND METHOD}

\section{A. Study Area}

Covered a total area of 8000 ha, Mursala Island is the largest island in the District of Central Tapanuli. It is located at the west coast of the province of North Sumatera $\left(01^{\circ} 35^{\prime} 15^{\prime \prime}-2^{\circ} 22^{\prime} 0^{\prime \prime} \mathrm{N}\right.$ and $\left.98^{\circ} 38^{\prime}-37^{\circ} 12^{\prime} \mathrm{E}\right)$, between Nias Island and the town of Sibolga (Fig. 1). Hilly areas dominate the island with the highest point of $500 \mathrm{~m}$ above sea level. The average temperature and humidity are $26.7^{\circ} \mathrm{C}$ and $75 \%$, respectively [8]. The average precipitation between 20092016 is $12.96 \mathrm{~mm} / \mathrm{year}$, with the average number of rainy days is 261 days/year [9].

\section{B. Field Survey}

To assess the population structure and estimate the population density of all Dipterocarpaceae species in Mursala Island, distance sampling using line-transect (Buckland et al. 2000) was used. A total of 8 line transects were purposively laid in the Island (Fig. 1) with the selection of the first point for each transect was merely based on the availability of a safe place for boot landing on the beach.

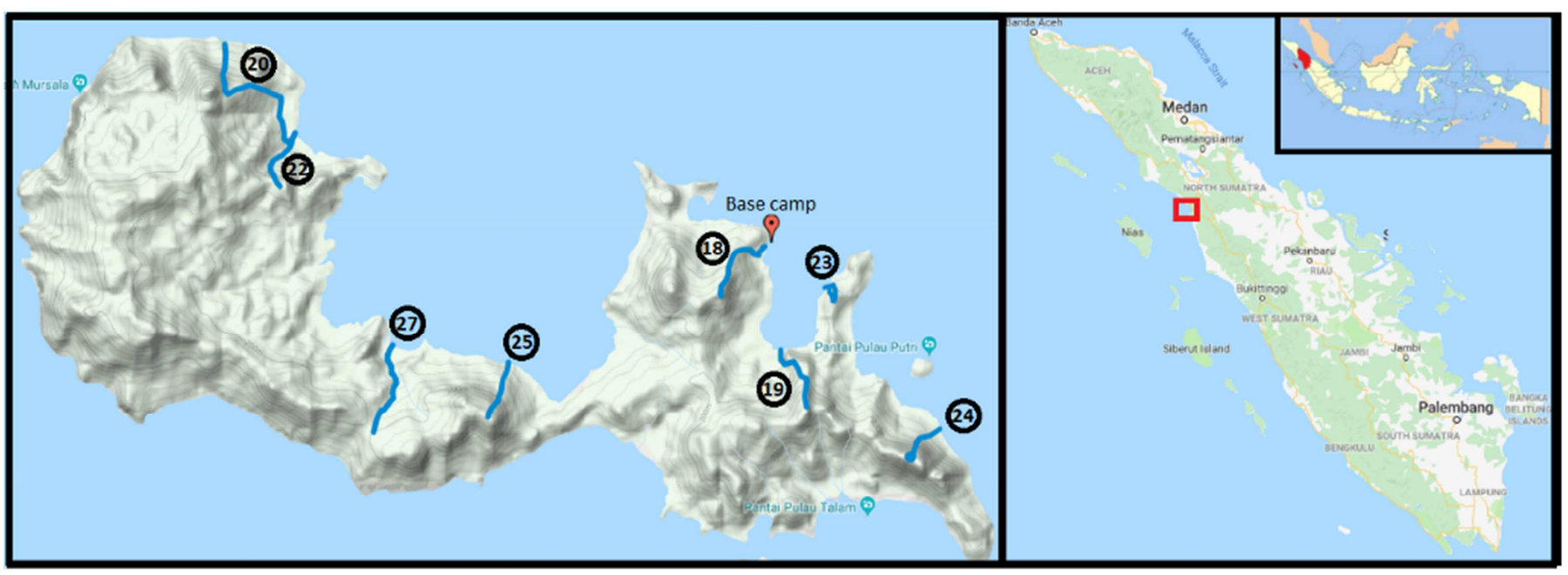

Fig. 1 Location of Mursala Island in District of Central Tapanuli, North Sumatra Province. Blue lines and its numbers are line transects used in the study

The length and elevation range of each transect were presented in Table 1.

TABLE I

Profile of Line TranseCtS USED IN MURSALA ISLAND

\begin{tabular}{|c|c|c|c|}
\hline No & Transect & Length $(\mathbf{k m})$ & Elevation \\
\hline 1 & 18 & 1.68 & $0-300$ \\
\hline 2 & 19 & 1.46 & $0-360$ \\
\hline 3 & 20 & 3.21 & $0-320$ \\
\hline 4 & 22 & 1.07 & $10-260$ \\
\hline 5 & 23 & 0.76 & $0-100$ \\
\hline 6 & 24 & 1.13 & $0-360$ \\
\hline 7 & 25 & 1.12 & $0-330$ \\
\hline 8 & 27 & 1.89 & $0-200$ \\
\hline
\end{tabular}

Along each transect, Dipterocarpaceae species were searched for by one assigned observer moving along the transect line. The perpendicular distance from the transect to each tree sighted was recorded using Laser Distance (Leica
Disto D2 NEW), and diameter at breast height (dbh) measurements were taken at $1.3 \mathrm{~m}$ above the ground using a diameter tape. Furthermore, the following environmental factors were also measured: elevation, slope, aspect, and terrain features (valley, slope, and ridge). For endemic species (D. cinereus and Hopea bancana), soil samples near the tree were collected to characterize the soil $\mathrm{pH}, \mathrm{C}, \mathrm{N}$ dan $\mathrm{P}$ content.

\section{Data Analysis}

The density of each species was estimated using Distance 7.1 Release 1 software [10]. Four model types (half-normal, hazard-rate, uniform, and negative-exponential), each with cosine, simple polynomial, and Hermite polynomial adjustments, were fitted to each population. The selection of the most appropriate detection function was based on the minimum Akaike Information Criterion (AIC) [11], [12].

\section{RESULTS AND DISCUSSION}

\section{A. Species Richness and Distribution}


Using a total of 8 line transects, 26 species of Dipterocarpaceae in Mursala Island were able to be identified. Genus that was represented by the highest number of species was Shorea (13 species), followed by Vatica (5), Dipterocarpus (4), Hopea (2), Cotylelobium (1), and Dryobalanops (1). Among these, two Mursala Island endemic species were identified: $D$. cinereus (Fig. 2) and $H$. bancana (Fig. 3). Furthermore, according to IUCN Red List Category, 11 species (42.3\%) were classified as critically endangered, four species $(15.4 \%)$ were of endangered, five species $(15.2 \%)$ were of vulnerable, one species was of near threatened, two species were of least concern and three species were not assessed yet.

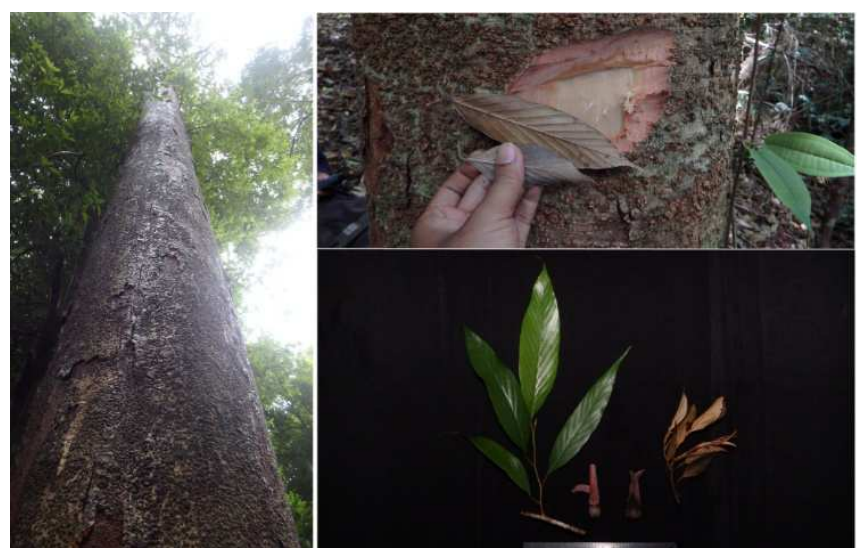

Fig. 2 Trunk, leaves and fruit morphology of Dipterocarpus cinereus.

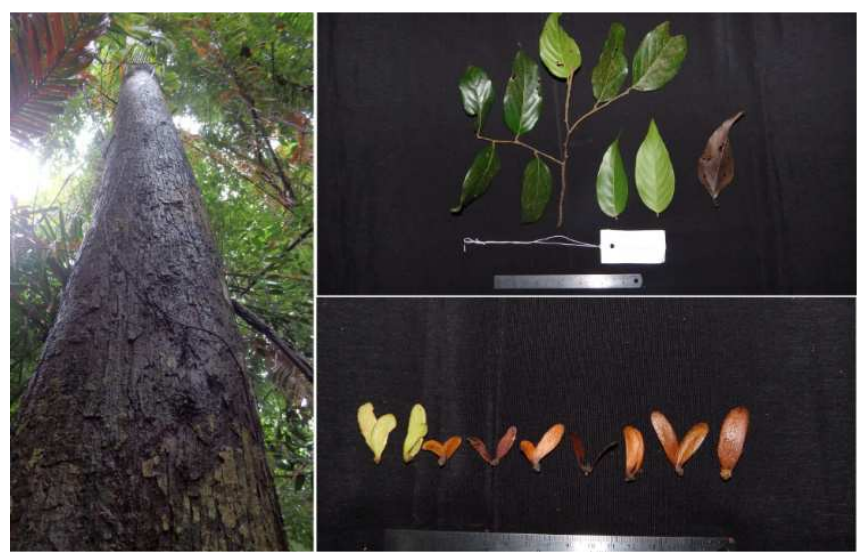

Fig. 3 Trunk, leaves and fruit morphology of Hopea bancana

The number of species identified in the present study was higher compared to of 22 species found by previous studies [6]-[8]. It was also higher compared to the number of 24 species recorded at the Herbarium Bogoriense. In addition, the Dipterocarpaceae diversity of the Island was much higher compared with other small islands around the mainland Sumatra. Siberut Island, for example, held 20 species of Dipterocarpaceae [13] and Enggano Island with only 5 species [14]. Furthermore, Lingga, Singkep, Batam, Bintan, and Bunguran of Riau Islands respectively had 13, 2, 7, 10, and 21 species [15].

Each species had its pattern of distribution on the island (Table 2). Shorea atrinervosa was species with the most widespread distribution. It was found at all eight linetransects used in the present study. The next species with widespread distribution were $D$. cinereus, $S$. acuminate, and $S$. multiflora, which were found at seven transects. On the contrary, there were species that have very narrow distribution on the island and were found only in one transect: S. johorensis, S. ochrophloia, S. palembanica, and Vatica perakensis.Population Structure

TABLE II

SPECIES OF DIPTEROCARPACEAE IDENTIFIED IN MURSALA ISLAND WITH ITS IUCN RED LIST STATUS, NUMBER OF OBSERVED INDIVIDUAL AND

DisTRIBUTION IN THE LINE TRANSECTS. NUMBERS OF THE LINE TRANSECT INSIDE THE BRACKETS REFERRED TO NUMBERS IN FIGURE 1

\begin{tabular}{|c|c|c|c|c|}
\hline No & Species & $\begin{array}{l}\text { Category of } \\
\text { IUCN Red } \\
\text { List }\end{array}$ & $\begin{array}{l}\text { Number of } \\
\text { observed } \\
\text { Individual } \\
\text { (mature } \\
\text { individual) }\end{array}$ & Line transect \\
\hline 1 & $\begin{array}{l}\text { Cotylelobium } \\
\text { melanoxylon }\end{array}$ & Endangered & $16(1)$ & $3(20,22,24)$ \\
\hline 2 & $\begin{array}{l}\text { Dipterocarpus } \\
\text { cinereus }\end{array}$ & $\begin{array}{l}\text { Critically } \\
\text { Endangered }\end{array}$ & $163(30)$ & $\begin{array}{l}7 \\
(18,19,20,22,24,25, \\
27)\end{array}$ \\
\hline 3 & $\begin{array}{l}\text { Dipterocarpus } \\
\text { cornutus }\end{array}$ & $\begin{array}{l}\text { Critically } \\
\text { Endangered }\end{array}$ & $65(24)$ & $5(20,23,24,25,27)$ \\
\hline 4 & $\begin{array}{l}\begin{array}{l}\text { Dipterocarpus } \\
\text { gracilis }\end{array} \\
\end{array}$ & Vulnerable & $11(1)$ & $2(22,27)$ \\
\hline 5 & $\begin{array}{l}\text { Dipterocarpus } \\
\text { kunstleri }\end{array}$ & $\begin{array}{l}\text { Critically } \\
\text { Endangered }\end{array}$ & $63(4)$ & $4(19,20,23,27)$ \\
\hline 6 & $\begin{array}{l}\text { Dryobalanops } \\
\text { sumatrensis }\end{array}$ & Vulnerable & $52(14)$ & $6(18,20,22,25,27)$ \\
\hline 7 & Hopea bancana & $\begin{array}{l}\text { Critically } \\
\text { Endangered } \\
\end{array}$ & $36(9)$ & $3(19,23,27)$ \\
\hline 8 & $\begin{array}{l}\text { Hopea } \\
\text { mengerawan }\end{array}$ & - & $4(1)$ & $2(22,25)$ \\
\hline 9 & $\begin{array}{l}\text { Shorea } \\
\text { acuminata }\end{array}$ & $\begin{array}{l}\text { Critically } \\
\text { Endangered }\end{array}$ & $101(60)$ & $\begin{array}{l}7 \\
(18,19,22,23,24,25, \\
27)\end{array}$ \\
\hline 10 & $\begin{array}{l}\text { Shorea } \\
\text { atrinervosa }\end{array}$ & Vulnerable & $160(52)$ & $\begin{array}{l}8 \\
(18,19,20,22,23,24, \\
25,27)\end{array}$ \\
\hline 11 & $\begin{array}{l}\text { Shorea } \\
\text { bracteolata }\end{array}$ & Endangered & $11(0)$ & $5(18,19,20,22,25)$ \\
\hline 12 & $\begin{array}{l}\text { Shorea } \\
\text { furfuracea }\end{array}$ & - & $15(4)$ & $3(9,25,27)$ \\
\hline 13 & $\begin{array}{l}\text { Shorea } \\
\text { johorensis }\end{array}$ & $\begin{array}{l}\text { Critically } \\
\text { Endangered }\end{array}$ & $5(4)$ & $1(20)$ \\
\hline 14 & Shorea lepidota & $\begin{array}{l}\text { Critically } \\
\text { Endangered }\end{array}$ & $84(40)$ & $4(20,22,25,27)$ \\
\hline 15 & $\begin{array}{l}\text { Shorea } \\
\text { leprosula }\end{array}$ & $\begin{array}{l}\text { Near } \\
\text { Threatened }\end{array}$ & $58(37)$ & $5(19,20,22,25,27)$ \\
\hline 16 & $\begin{array}{l}\text { Shorea } \\
\text { multiflora }\end{array}$ & $\begin{array}{l}\text { Least } \\
\text { Concern }\end{array}$ & $228(62)$ & $\begin{array}{l}7 \\
(19,18,22,23,24,25, \\
27)\end{array}$ \\
\hline 17 & $\begin{array}{l}\text { Shorea } \\
\text { ochrophloia }\end{array}$ & $\begin{array}{l}\text { Critically } \\
\text { Endangered }\end{array}$ & $4(0)$ & $1(27)$ \\
\hline 18 & Shorea ovata & Endangered & $36(20)$ & $5(20,22,24,25,27)$ \\
\hline 19 & $\begin{array}{l}\begin{array}{l}\text { Shorea } \\
\text { palembanica }\end{array} \\
\end{array}$ & $\begin{array}{l}\text { Critically } \\
\text { Endangered }\end{array}$ & $2(1)$ & $1(27)$ \\
\hline 20 & $\begin{array}{l}\text { Shorea } \\
\text { parvifolia }\end{array}$ & $\begin{array}{l}\text { Least } \\
\text { Concern }\end{array}$ & $20(8)$ & $5(18,20,22,25,27)$ \\
\hline 21 & $\begin{array}{l}\text { Shorea } \\
\text { retinodes }\end{array}$ & - & $16(10)$ & $4(20,22,25,27)$ \\
\hline 22 & $\begin{array}{l}\text { Vatica } \\
\text { pauciflora }\end{array}$ & Vulnerable & $5(0)$ & $2(20,27)$ \\
\hline 23 & $\begin{array}{l}\text { Vatica } \\
\text { perakensis }\end{array}$ & Endangered & $2(1)$ & $1(22)$ \\
\hline 24 & $\begin{array}{l}\text { Vatica } \\
\text { soepadmoi }\end{array}$ & $\begin{array}{l}\text { Critically } \\
\text { Endangered }\end{array}$ & $5(0)$ & $4(18,19,25,27)$ \\
\hline 25 & $\begin{array}{l}\text { Vatica } \\
\text { stapfiana }\end{array}$ & Vulnerable & $5(1)$ & $2(20,23)$ \\
\hline 26 & $\begin{array}{l}\text { Vatica venulosa } \\
\text { ssp. } \\
\text { Simalurensis }\end{array}$ & $\begin{array}{l}\text { Critically } \\
\text { Endangered }\end{array}$ & $120(4)$ & $5(18,19,22,23,24)$ \\
\hline
\end{tabular}

During the survey, a total of 1,289 individual trees of Dipterocarpaceae were observed (Table 2). Species with the highest number of observed individuals was S. multiflora (228 individuals), followed by $D$. cinereus (163), $S$. 
atrinervosa (160), and Vatica venulosa ssp. simalurensis (120), whereas species with the lowest number of observed individuals were $S$. palembanica and $V$. perakensis with only two individuals each.

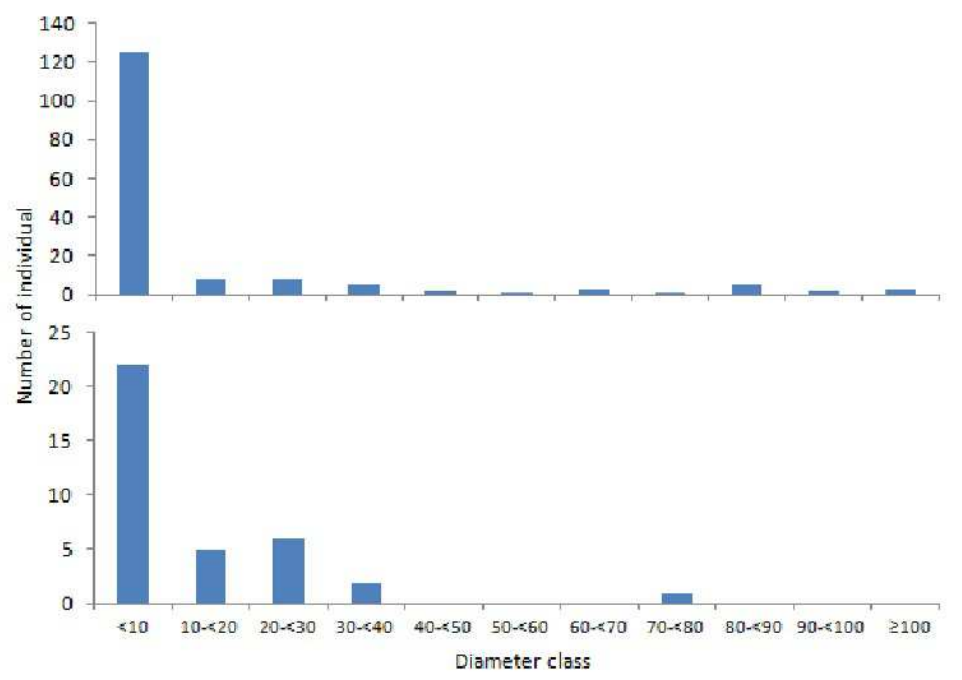

Fig. 4 Population structures of endemic Dipterocarpus cinereus (top) and Hopea bancana (bottom) based on diameter classes.

Based on dbh distribution, population structures of all species were dominantly characterized by the absence or a deficient number of large-diameter individuals. They thus were deviated from the standard inverted-J type curves commonly found in tropical forests. This pattern of dbh distribution suggested an unhealthy population condition. The pattern was similar for endemic (Fig. 4) and nonendemic species (Fig. 5). The most likely reason behind this was the observed illegal logging activities in the island. As the logger targeted mature Dipterocarpaceae individuals, their number in the population became very low or even vanish. Similar deviation of dbh distribution from normal reverse-J shape due to selective illegal logging was also observed in the Kibauni Hill Forest, Kenya [16], the forest of Tripura, India [17], forest of Bago Yoma, Myanmar [18], and Ndege Forest Reserve, Tanzania [19].

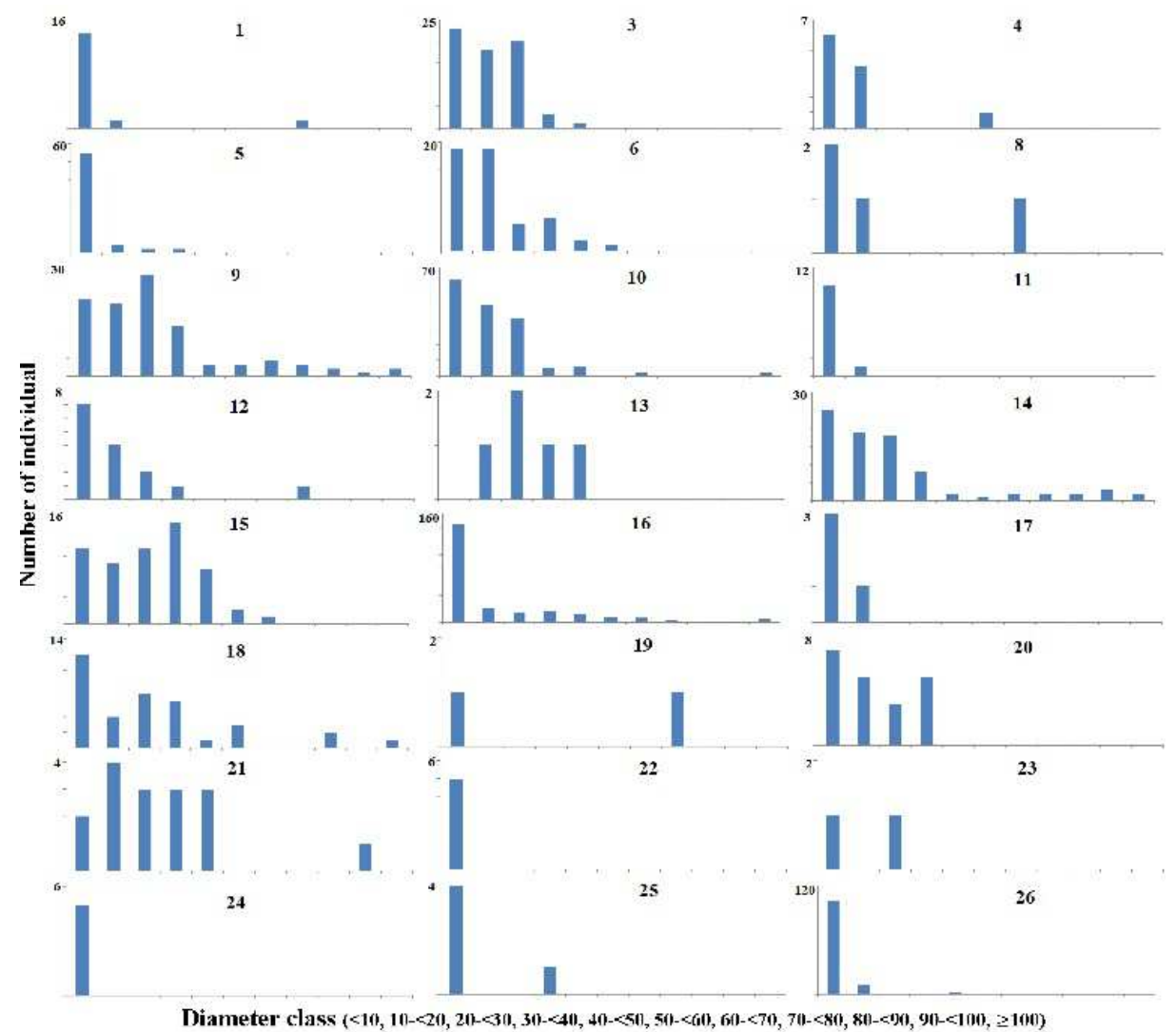

Fig. 5 Population structures of Dipterocarpaceae species in Mursala Island based on diameter classes. Number on each graph referred to the species number in Table 2 


\section{B. Species Density}

Based on distance analysis, the Hazard-rate model (with Hermite series adjustment) was found most suitable for most populations, as judged by low AIC (Table 3). The species with the highest individual density was $S$. multiflora
(21.72 \pm 10.96 individual/ha), whereas the species with the lowest individual density was $S$. johorensis $(0.06 \pm 0.01$ individual/ha). The density of endemic tree $D$. cinereus and $H$. bancana were $8.4 \pm 3.79$ individual/ha and $3.48 \pm 2.78$ individual/ha, respectively.

TABLE III

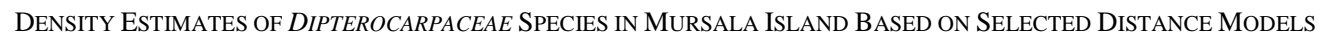

\begin{tabular}{|c|c|c|c|c|c|}
\hline No & Species & $\begin{array}{l}\text { Density estimate } \\
\text { (individuals/ha) }\end{array}$ & Model/Series & $\begin{array}{l}\text { Akaike } \\
\text { Information } \\
\text { Criterion }\end{array}$ & $\begin{array}{l}95 \% \text { Confidence } \\
\text { interval }\end{array}$ \\
\hline 1 & Cotylelobium melanoxylon & $1.18 \pm 1.12$ & Hazard Rate/Cosine & 78.29 & $0.20-6.96$ \\
\hline 2 & Dipterocarpus cinereus & $8.4 \pm 3.79$ & Hazard Rate/Cosine & 848.03 & $3.08-22.94$ \\
\hline 3 & Dipterocarpus cornutus & $2.77 \pm 1.27$ & Hazard Rate/Polynomial & 397.88 & $1.03-7.41$ \\
\hline 4 & Dipterocarpus gracilis & $0.59 \pm 0.48$ & Negative Exponential/Hermite & 63.26 & $0.12-2.86$ \\
\hline 5 & Dipterocarpus kunstleri & $4.12 \pm 3.78$ & Hazard rate/Hermite & 289.29 & $0.68-25.7$ \\
\hline 6 & Dryobalanops sumatrensis & $2.23 \pm 0.94$ & Negative Exponential/Hermite & 301.28 & $0.92-5.44$ \\
\hline 7 & Hopea bancana & $3.48 \pm 2.78$ & Hazard rate/Hermite & 149.1 & $0.7-17.24$ \\
\hline 8 & Hopea mengerawan & $0.19 \pm 0.15$ & Uniform/Hermite & 17.21 & $0.03-1.04$ \\
\hline 9 & Shorea acuminata & $3.89 \pm 1.55$ & Hazard rate/Hermite & 551.13 & $1.59-9.53$ \\
\hline 10 & Shorea atrinervosa & $9.43 \pm 4.55$ & Hazard rate/Hermite & 897.39 & $3.27-27.17$ \\
\hline 11 & Shorea bracteolata & $1.2 \pm 0.69$ & Hazard rate/Hermite & 45.49 & $0.38-3.79$ \\
\hline 12 & Shorea furfuracea & $1.26 \pm 0.89$ & Hazard Rate/Polynomial & 76.92 & $0.33-4.84$ \\
\hline 13 & Shorea johorensis & $0.06 \pm 0.01$ & Uniform/Cosine & 28.9 & 0.03-0.09 \\
\hline 14 & Shorea lepidota & $4.31 \pm 1.89$ & Hazard Rate/Cosine & 470.25 & $1.64-11.34$ \\
\hline 15 & Shorea leprosula & $2.11 \pm 1.04$ & Hazard rate/Hermite & 356.48 & $0.73-6.16$ \\
\hline 16 & Shorea multiflora & $21.72 \pm 10.96$ & Hazard Rate/Cosine & 966.68 & $7.47-63.15$ \\
\hline 17 & Shorea ochrophloia & $0.14 \pm 0.06$ & Uniform/Hermite & 16.64 & $0.06-0.33$ \\
\hline 18 & Shorea ovata & $0.33 \pm 0.04$ & Uniform/Cosine & 198.39 & $0.26-0.42$ \\
\hline 19 & Shorea palembanica & $0.22 \pm 0.14$ & Uniform/Polynomial & 4.65 & $0.06-0.85$ \\
\hline 20 & Shorea parvifolia & $1.88 \pm 1.33$ & Hazard rate/Hermite & 97.39 & $0.59-6$ \\
\hline 21 & Shorea retinodes & $0.68 \pm 0.35$ & Uniform/Cosine & 93.44 & $0.24-1.92$ \\
\hline 22 & Vatica pauciflora & $0.47 \pm 0.11$ & Uniform/Hermite & 9.56 & $0.28-0.78$ \\
\hline 23 & Vatica perakensis & $0.14 \pm 0.13$ & Uniform/Hermite & 7.17 & $0.02-1.05$ \\
\hline 24 & Vatica soepadmoi & $0.41 \pm 0.17$ & Uniform/Cosine & 16.09 & $0.15-1.1$ \\
\hline 25 & Vatica stapfiana & $0.38 \pm 0.13$ & Uniform/Polynomial & 13.86 & $0.17-0.79$ \\
\hline 26 & Vatica venulosa ssp. Simalurensis & $9.01 \pm 5.65$ & Hazard Rate/Polynomial & 501.88 & $2.36-34.37$ \\
\hline
\end{tabular}

Distance sampling has many advantages than other methods, including more economical, statistically defensible, and more practical in quantitating plant species characteristics [20]. The present study showed that distance sampling using line-transect could be effectively used to survey the population status and estimate the species density of Dipterocarpaceae species in Mursala Island. The method, for instance, could detect 30 mature individuals of the endemic tree $D$. cinereus. This number was much higher compared to 3 mature individuals observed by previous studies. From these observed individuals, the density of $D$. cinereus was estimated to be $8.4 \pm 3.79$ individuals/ha. This density estimate (and the estimate of the other species), however, had to be used carefully when applying it to other un-surveyed areas on the island. Habitat specificity as well as the high rate of tree cutting and land conversion, might make the density estimate in other areas become unreliable.

\section{Habitat Characteristics}

Dipterocarpaceae species were found throughout the island from near seal level at an elevation of $5 \mathrm{~m}$ to the highest peak at $500 \mathrm{~m}$ above sea level. However, most of the individuals were found on ridge and slope terrains at a higher elevation, including the endemic $D$. cinereus that was dominantly located at the top of hills. There were only a few species found on valleys of the Island, such as D. cornutus, Dryobalanops sumatrensis, and S. johorensis (Table 4). 
TABLE IV

HABITAT CHARACTERISTICS OF DIPTEROCARPACEAE SPECIES IN MURSALA ISLAND

\begin{tabular}{|c|c|c|c|c|c|c|c|c|c|}
\hline \multirow{2}{*}{ No } & \multirow{2}{*}{ Species } & \multicolumn{2}{|c|}{ Slope } & \multicolumn{2}{|c|}{ Elevation } & \multicolumn{2}{|r|}{ Aspect } & \multicolumn{2}{|r|}{ Terrain } \\
\hline & & Mode & Range & Mode & Range & Mode & Range & Mode & Range \\
\hline 1 & $\begin{array}{l}\text { Cotylelobium } \\
\text { melanoxylon }\end{array}$ & 21 & $4-30$ & 258 & $250-335$ & West & Northeast, West & Ridge & Slope-Ridge \\
\hline 2 & Dipterocarpus cinereus & 30 & $10-36$ & 340 & $19-371$ & Northeast & $\begin{array}{l}\text { North-Southeast, } \\
\text { Southwest-West }\end{array}$ & Slope & Slope-Ridge \\
\hline 3 & Dipterocarpus cornutus & 10 & $0-35$ & 55 & $12-185$ & Northeast & West-Northeast, flat & Valley & $\begin{array}{l}\text { Valley-slope- } \\
\text { ridge }\end{array}$ \\
\hline 4 & Dipterocarpus gracilis & 14 & $10-24$ & 98 & $49-164$ & East & Northeast-East & Ridge & Slope-Ridge \\
\hline 5 & Dipterocarpus kunstleri & 12 & $4-32$ & 97 & $26-162$ & Northeast & West-Southeast & Slope & Slope-Ridge \\
\hline 6 & $\begin{array}{l}\text { Dryobalanops } \\
\text { sumatrensis }\end{array}$ & 21 & $0-35$ & 6 & $5-258$ & North & $\begin{array}{l}\text { North-East, South, } \\
\text { West, Flat }\end{array}$ & Valley & $\begin{array}{l}\text { Valley-slope- } \\
\text { ridge }\end{array}$ \\
\hline 7 & Hopea bancana & 2 & $2-35$ & 13 & $12-190$ & Northwest & West-East & Slope & Slope-Ridge \\
\hline 8 & Hopea mengerawan & 12 & $12-15$ & 169 & $150-169$ & Northwest & Northwest, Flat & Ridge & Ridge \\
\hline 9 & Shorea acuminata & 12 & $4-55$ & 317 & $57-370$ & Northeast & North-West, Flat & Slope & Slope-Ridge \\
\hline 10 & Shorea atrinervosa & 21 & $4-57$ & 259 & $20-344$ & West & $\begin{array}{l}\text { North- } \\
\text { East,Southwest, } \\
\text { Northwest, Flat }\end{array}$ & Ridge & $\begin{array}{l}\text { Valley-slope- } \\
\text { ridge }\end{array}$ \\
\hline 11 & Shorea bracteolata & 28 & $5-32$ & 180 & $41-264$ & North & North-East, West, Flat & Ridge & $\begin{array}{l}\text { Valley-slope- } \\
\text { ridge }\end{array}$ \\
\hline 12 & Shorea furfuracea & 33 & $10-35$ & 295 & $22-342$ & North & West-East & Slope & $\begin{array}{l}\text { Valley-slope- } \\
\text { ridge }\end{array}$ \\
\hline 13 & Shorea johorensis & 0 & $0-31$ & 19 & $18-120$ & Flat & East, Flat & Valley & Valley-Slope \\
\hline 14 & Shorea lepidota & 10 & $4-36$ & 140 & $61-365$ & North & West-East, Flat & Slope & Slope-Ridge \\
\hline 15 & Shorea leprosula & 30 & $0-32$ & 75 & $12-312$ & East & $\begin{array}{l}\text { Northwest-Southeast, } \\
\text { Flat }\end{array}$ & Ridge & $\begin{array}{l}\text { Valley-slope- } \\
\text { ridge }\end{array}$ \\
\hline 16 & Shorea multiflora & 28 & $4-57$ & 108 & $53-338$ & North & All & Ridge & Slope-Ridge \\
\hline 17 & Shorea ochrophloia & 6 & $6-35$ & 67 & $57-160$ & Northwest & West-Northeast, East & Slope & Slope \\
\hline 18 & Shorea ovata & 31 & $4-55$ & 160 & $29-370$ & Northeast & $\begin{array}{l}\text { North-Northeast, } \\
\text { West, Flat }\end{array}$ & Slope & Slope-Ridge \\
\hline 19 & Shorea palembanica & - & $24-15$ & - & $167-170$ & - & East & - & Slope \\
\hline 20 & Shorea parvifolia & 31 & $6-33$ & 353 & $95-353$ & Northeast & $\begin{array}{l}\text { North-East, } \\
\text { Northwest }\end{array}$ & Ridge & Slope-Ridge \\
\hline 21 & Shorea retinodes & 6 & $6-28$ & 66 & $12-172$ & Northwest & $\begin{array}{l}\text { North-East, } \\
\text { Northwest, Flat }\end{array}$ & Slope & $\begin{array}{l}\text { Valley-slope- } \\
\text { ridge }\end{array}$ \\
\hline 22 & Vatica pauciflora & - & $12-21$ & - & $10-294$ & - & North-East & Slope & Valley-Slope \\
\hline 23 & Vatica perakensis & - & $20-27$ & - & $127-200$ & - & Northeast, Northwest & - & Ridge \\
\hline 24 & Vatica soepadmoi & 12 & $5-18$ & - & $108-341$ & North & North-Northeast & Ridge & Slope-Ridge \\
\hline 25 & Vatica stapfiana & 4 & $4-30$ & 120 & $5-312$ & - & East-Southeast, West & Ridge & $\begin{array}{l}\text { Valley-slope- } \\
\text { ridge }\end{array}$ \\
\hline 26 & $\begin{array}{l}\text { Vatica venulosa ssp. } \\
\text { Simalurensis }\end{array}$ & 55 & $4-55$ & 273 & $70-338$ & North & Southeast-Northeast & Ridge & Slope-Ridge \\
\hline
\end{tabular}

Furthermore, the North aspect slope (including Northeast and Northwest) was observed to be the most favorable habitat for the Dipterocarpaceae species. For the endemic $D$. cinereus, its soil was characterized by $\mathrm{pH}$, organic carbon, nitrogen and phosphorus content of, respectively, 4.72 \pm 0.15 , $5.92 \pm 1.48 \%, 0.34 \pm 0.02 \%$, and $6.67 \pm 1.69 \mathrm{ppm}$, whereas soil $\mathrm{pH}$, organic carbon, nitrogen and phosphorus content of $H$. bancana was $5.37 \pm 0.27,2.84 \pm 0.13 \%, 0.2 \pm 0.02 \%$, and $9.33 \pm 5.84 \mathrm{ppm}$, respectively.

\section{Threats and Conservation Actions}

Two main threats for Dipterocarpaceae species in Mursala Island were identified during the field survey: heavy illegal logging and forest conversion. For the first threat, there are two to four sources of chainsaw sound that were heard by the team every day during the data collection. Local people did the logging activities, and they targeted mainly big trees from Dipterocarpaceae species, including the endemic tree $D$. cinereus. In addition, many logging sites (Fig. 6a-c) were found, including tracks to transport the processed timber from the logging site to the beach (Fig. 6d). After reaching the beach, the processed timbers were covered by twig and branch of trees (Fig. 6e-f) in order to hide the woods from the marine police patrol. In addition to trading in the main island of Sumatra, the timbers were also 

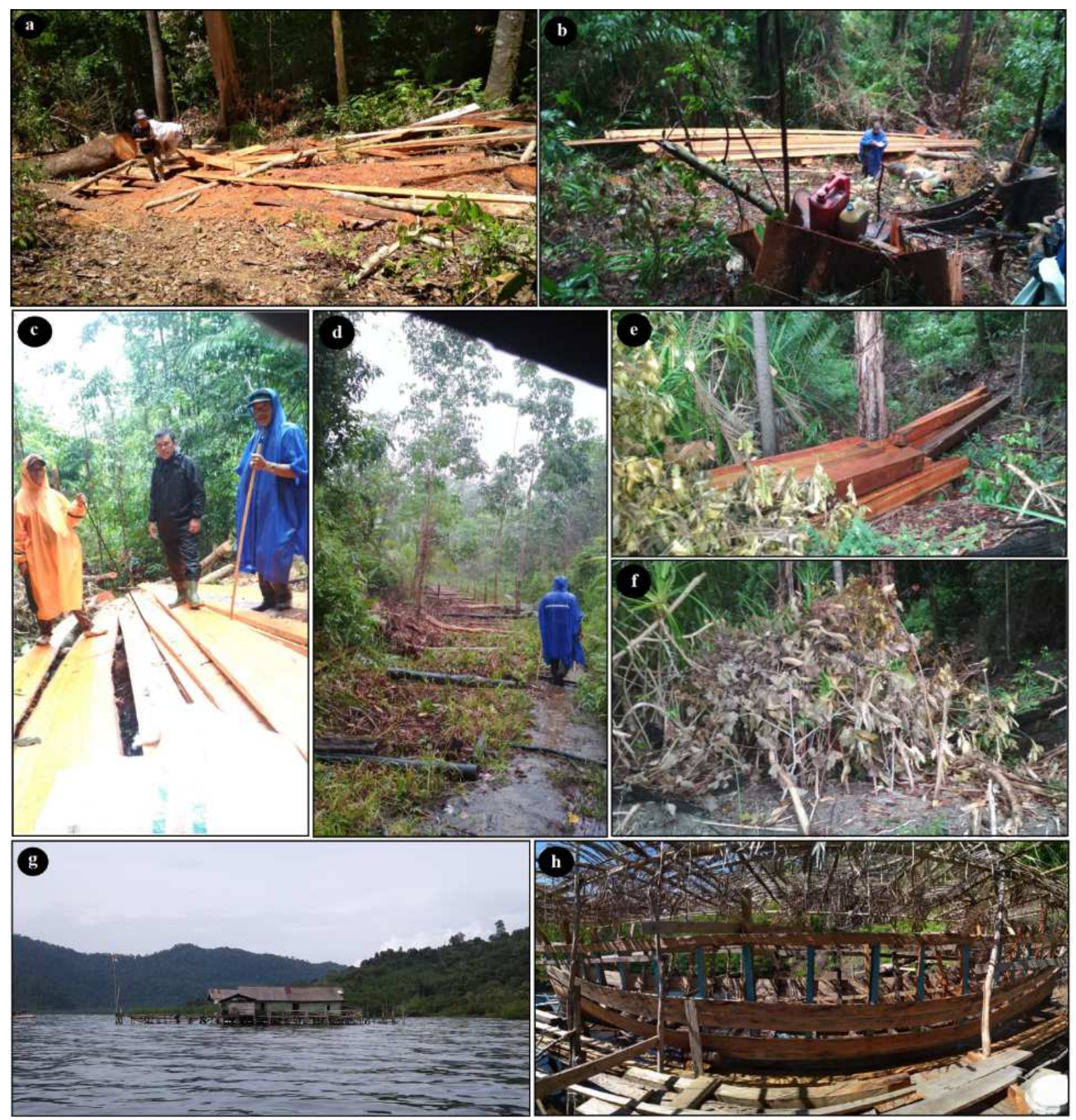

Fig. 6 Illegal logging activities found in Mursala Island: a-c) logging sites, d) tract for transporting processed timber in the forest, e-f) processed timber in the beach ready for transporting, g) house made of timber cut from the forest, h) boat made of timber from the forest.

The second threat to the Dipterocarpaceae was habitat conversion. Local people cut the forest along the coastal areas and plant the rubber tree, cassava, sweet potato, and others. These plantations were distributed mainly along the northern beach of the Island and could stretch up to more than $100 \mathrm{~m}$ into the forest areas.

Mursala Island could be considered as one of the important areas for Dipterocarpaceae conservation. Among 26 recorded species, $20(76.9 \%)$ of them were threatened species according to the IUCN Red List category and criteria.
Furthermore, Mursala Island was the only habitat for $D$. cinereus, the previously reported extinct tree species. Moreover, for $H$. bancana, although it was believed to be endemic to Sumatra [21], the present study and the collections records of Bogor Botanic Gardens revealed that Mursala Island was the only remaining habitat for the species. Thus, comprehensive conservation actions are needed to protect the Dipterocarpaceae diversity in the Island. Increasing the protection level of the island and providing alternative sources of living for the illegal logger 
through commercialization of non-timber forest products, ecotourism, and sustainable fishery are some of the recommended actions. These actions must be implemented immediately as the present study showed timber extraction activities are still undergoing on the island at an alarming rate.

\section{CONCLUSION}

Despite extensive wood extraction that has been undergoing for a long time, Mursala Island still possesses a high species diversity of Dipterocarpaceae. This high species diversity has made the island one of the hotspots for Dipterocarpaceae biodiversity. Immediate comprehensive conservation actions need to be implemented to conserve the species and their habitat.

\section{ACKNOWLEDGMENT}

This work was undertaken with the support of The Mohamed bin Zayed Species Conservation Fund (Project no. 182517815) and Global Tree Campaign (http://globaltrees.org/). We thank to Bpk Rahmat, Bpk Pasaribu, Bpk Sumarno, Farhan, Bang Edi, Ucok and Bpk Sindoro for field assistance. We would also like to thank Bpk. Asep Ferry MA from KPH Unit XI Pandan for the research permit.

\section{REFERENCES}

[1] Purwaningsih, "Sebaran ekologi jenis-jenis Dipterocarpaceae di Indonesia” Biodiversitas, vol. 5, pp. 89-95, Jul. 2014.

[2] G. Maury-Lechon and L. Curtet, "Biogeography and evolutionary systematics of Dipterocarpaceae" in Review of Dipterocarps: Taxonomy, ecology and silviculture, S. Appanah and J.M. Turnbull, Eds. Bogor, Indonesia: A Center for International Forestry Research, 1998.

[3] R. Hegnauer. (2020) Dipterocarpaceae. [Online]. Available: http://portal.cybertaxonomy.org/flora-malesiana/node/4361

[4] IUCN. (2019) The IUCN Red List of Threatened Species. Version 2019-1. [Online]. Available: http://www.iucnredlist.org

[5] P. Ashton. (1998) Dipterocarpus cinereus. The IUCN Red List of Threatened Species 1998: e.T33374A9773444. [Online]. Available: http://dx.doi.org/10.2305/IUCN.UK.1998.RLTS.T33374A9773444.e n.

[6] Y. W.C. Kusuma, Wihermanto, and R. A. Risna, "Rediscovery of the supposedly extinct Dipterocarpus cinereus" Oryx, vol. 47, pp. 324, Jul. 2013.

[7] H.H. Rachmat and A. Subiakto, "Conserving the previously reported extinct tree species Dipterocarpus cinereus: An ex-situ approach for the species conservation strategy" in Prosiding Seminar Nasional Masyarakat Biodiversitas Indonesia, 2015, pp. 560-564.
[8] H. H. Rachmat, A. Subiakto, K. Wijaya, and A. Susilowati, "Alarming call from Mursala Island, North Sumatra, Indonesia: The urgent task of conserving the previously reported extinct of Dipterocarpus cinereus" Biodiversitas, vol. 19, pp. 399-405, Mar. 2018.

[9] BPS Kabupaten Tapanuli Tengah, Kabupaten Tapanuli Tengah Dalam Angka 2017. Tapanuli Tengah, Indonesia: BPS Kabupaten Tapanuli Tengah, 2017.

[10] Thomas, S. T. Buckland, E. A. Rexstad, J. L. Laake, S. Strindberg, S. L. Hedley, J. R. B. Bishop, T.A. Marques, and K. P. Burnham, "Distance software: Design and analysis of distance sampling surveys for estimating population size" Journal of Applied Ecology, vol. 47, pp. 5-14, Feb. 2010.

[11] H. Akaike "Information Theory and an Extension of the Maximum Likelihood Principle" in International Symposium on Information Theory 2nd Edition, B. N. Petran and F. Csaaki, Eds. Budapest, Hungary: Akadèemiai Kiadi, 1973.

[12] K.P. Burnham and D. R. Anderson, Model Selection and MultiModel Inference: a Practical Information-Theoretic Approach. New York, USA: Springer-Verlag, 2002.

[13] TFCA-Sumatera. (2016) TN Siberut dan Kep Mentawai. [Online]. Available: http://tfcasumatera.org/tn-siberut-dan-kep-mentawai/

[14] I. Maryanto, A. Hamidy, A. P. Keim, V. B. L. Sihotang, P. Lupiyaningdyah, M. Irham and M. Ardiyani, Ekspedisi Pulau Enggano. Jakarta, Indonesia: LIPI Press, 2017.

[15] A. Subiakto and H. H. Rachmat, "Exploration, Collection, and Conservation of Dipterocarps in Riau Islands" in Prosiding Seminar Nasional Masyarakat Biodiversitas Indonesia, 2015, pp. 428-433.

[16] V. Mainga, W. Musila, J. Kimeu, C. Chesire and J. Kariuki. (2012) Kibauni Hill Forest Plant Assessment: Floristic Composition, Structure and Conservation Status. Final Report of the Rufford small grants for Nature. [Online]. Available: https://www.rufford.org/files/9825$1 \% 20$ Detailed\%20Final\%20Report.pdf

[17] K. Majumdar and B. K. Datta, "Effects of Anthropogenic Disturbances on Vegetation Diversity and Structure: a Case Study in the Remnant Forests Surrounding the Village Ecosystems of Tripura, Northeast India" Chinese Journal of Population Resources and Environment, vol. 13, pp. 332-340, Aug. 2015.

[18] T. C. Khai, N. Mizoue, T. Kajisa, T. Ota, and S. Yoshida, "Stand structure, composition and illegal logging in selectively logged production forests of Myanmar: Comparison of two compartments subject to different cutting frequency" Global ecology and conservation, vol. 7, pp. 132-140, Jul. 2016.

[19] R. M. Byamungu, S. B. Mariki and N. P. Mkiramweni, "Tree stock, structure and use of common woody species of a town neighboring forest reserve in Tanzania: Implication for managing carbon accumulation" in Forest Biomass and Carbon, 2018, G. Shukla and S. Chakravarty, Eds. London, UK: IntechOpen.

[20] M. Mirzaei and A. E. Bonyad, "Comparison of fixed area and distance sampling methods in open forests: Case study of Zagros forest, Iran" Journal of forestry research, vol. 27, pp. 1121-1126, Apr. 2016.

[21] P. Ashton. (1998) Hopea bancana. The IUCN Red List of Threatened Species 1998: e.T33382A9774710. [Online]. Available: http://dx.doi.org/10.2305/IUCN.UK.1998.RLTS.T33382A9774710.e 